\title{
PTBP1 Genetic Variants Affect the Clinical Response to Androgen-deprivation Therapy in Patients With Prostate Cancer
}

\author{
SHU-PIN HUANG ${ }^{1,2,3,4^{*}}$, LIH-CHYANG CHEN ${ }^{5 *}$, YEI-TSUNG CHEN ${ }^{6}$, CHENG-HSUEH LEE $^{1,2}$, \\ CHAO-YUAN HUANG ${ }^{7}$, CHIA-CHENG YU ${ }^{8,9,10}$, VICTOR C. LIN ${ }^{11,12}$, TE-LING LU ${ }^{13}$ and BO-YING BAO ${ }^{13,14,15}$ \\ ${ }^{1}$ Department of Urology, Kaohsiung Medical University Hospital, Kaohsiung, Taiwan, R.O.C.; \\ ${ }^{2}$ Graduate Institute of Clinical Medicine, College of Medicine, \\ Kaohsiung Medical University, Kaohsiung, Taiwan, R.O.C.; \\ ${ }^{3}$ Department of Urology, Faculty of Medicine, College of Medicine, \\ Kaohsiung Medical University, Kaohsiung, Taiwan, R.O.C.; \\ ${ }^{4}$ Center for Cancer Research, Kaohsiung Medical University, Kaohsiung, Taiwan, R.O.C.; \\ ${ }^{5}$ Department of Medicine, Mackay Medical College, New Taipei City, Taiwan, R.O.C.; \\ ${ }^{6}$ Department of Life Sciences and Institute of Genome Sciences, National Yang-Ming University, Taipei, Taiwan, R.O.C.; \\ ${ }^{7}$ Department of Urology, National Taiwan University Hospital, \\ College of Medicine, National Taiwan University, Taipei, Taiwan, R.O.C.; \\ ${ }^{8}$ Division of Urology, Department of Surgery, Kaohsiung Veterans General Hospital, Kaohsiung, Taiwan, R.O.C.; \\ ${ }^{9}$ Department of Urology, School of Medicine, National Yang-Ming University, Taipei, Taiwan, R.O.C.; \\ ${ }^{10}$ Department of Pharmacy, College of Pharmacy and Health Care, Tajen University, Pingtung, Taiwan, R.O.C.; \\ ${ }^{11}$ Department of Urology, E-Da Hospital, Kaohsiung, Taiwan, R.O.C.; \\ ${ }^{12}$ School of Medicine for International Students, I-Shou University, Kaohsiung, Taiwan, R.O.C.; \\ ${ }^{13}$ Department of Pharmacy, China Medical University, Taichung, Taiwan, R.O.C.; \\ ${ }^{14}$ Sex Hormone Research Center, China Medical University Hospital, Taichung, Taiwan, R.O.C.; \\ ${ }^{15}$ Department of Nursing, Asia University, Taichung, Taiwan, R.O.C.
}

\begin{abstract}
Background/Aim: Heterogeneous nuclear ribonucleoproteins ( $h n R N P S$ ) contribute to multiple cellular functions including RNA splicing, stabilization, transcriptional and translational regulation, and signal transduction. However, the prognostic importance of genetic variants of hnRNP genes in clinical outcomes of prostate cancer remains to be elucidated. Patients and Methods: We studied the association of 78 germline single-nucleotide polymorphisms (SNPs) in 23 hnRNP genes with the overall survival (OS), cancer-specific
\end{abstract}

This article is freely accessible online.

*These Authors contributed equally to this work.

Correspondence to: Bo-Ying Bao, Department of Pharmacy, China Medical University, 100 Jingmao Road Section 1, Beitun District, Taichung 406, Taiwan, R.O.C. Tel: +886 422053366 ext. 5126, Fax: +886 422031075; E-mail: bao@mail.cmu.edu.tw

Key Words: Androgen-deprivation therapy, heterogeneous nuclear ribonucleoprotein, meta-analysis, prostate cancer, $P T B P 1$, survival. survival (CSS), and progression-free survival (PFS) in 630 patients with prostate cancer receiving androgen-deprivation therapy (ADT). Results: PTBP1 rs10420407 was the most significant SNP (false discovery rate $q=0.003$ ) and carriers of the A allele exhibited poor OS, CSS, and PFS. Multivariate Cox analysis confirmed PTBP1 rs10420407 A allele was an independent negative prognostic factor for OS and PFS. Expression quantitative trait loci analysis showed that the rs 10420407 A allele had a trend towards increased PTBPI $m R N A$ expression, and higher expression was correlated with prostate cancer aggressiveness and poor patient prognosis. Meta-analysis of 16 independent studies further indicated a tumorigenic effect of $P T B P 1$, with a higher expression in prostate cancers than in adjacent normal tissues $(p<0.001)$. Conclusion: Our data suggest that PTBP1 rs10420407 may influence patient response to ADT, and PTBP1 may be involved in the pathogenesis of prostate cancer progression.

Prostate cancer is the second-most common cancer affecting men worldwide, with 1.4 million new cases in 2020 (1). Since androgen signalling affects the proliferation and 
differentiation of the normal prostate epithelium, and stimulates prostate cancer cell growth, androgen-deprivation therapy (ADT) is used as a common and effective treatment for patients with advanced prostate cancer (2). However, the disease inevitably progresses to castration-resistant prostate cancer (CRPC) despite continuous hormonal regulation; patients with metastatic CRPC have a poor prognosis with a median survival time of 16-18 months (3). Genetic and environmental factors are believed to contribute to the development of prostate cancer; a risk of developing the disease increases with age, having an African ancestry, North American or northern European location, and positive family history (4). A recent study estimated that $57 \%$ of the variability in prostate cancer risk is attributed to genetic factors, and it is recognised as one of the most heritable cancers (5). The variations in critical genes of individuals may explain the variable patient outcomes, and the use of this information may help determine the treatment response, and guide optimal dose or patient selection.

Heterogeneous nuclear ribonucleoproteins (hnRNPs) are a family of proteins comprising more than 20 major RNAbinding proteins that are responsible for post-transcriptional control of RNA, such as pre-mRNA splicing and polyadenylation, and functional mRNA export, turnover, and translation (6). The amino terminus of hnRNPs contains an RNA recognition motif that binds to pre-mRNA sequences, and the carboxyl terminus contains a glycine-rich domain that interacts with other hnRNPs (7). The hnRNPs generally bind to exonic and intronic splicing silencers to inhibit or promote splicing reactions by regulating the assembly of the spliceosome complex or the recruitment of serine/argininerich proteins to the splicing sites (8). Recent studies demonstrated that the expression of hnRNPs is commonly deregulated in multiple cancer types including prostate cancer. HNRNPA1, HNRNPA2, and polypyrimidine tract binding protein 1 (PTBP1) have been demonstrated to be up-regulated by the MYC proto-oncogene, which induces the production of pyruvate kinase to activate aerobic glycolysis in cancer cells (9). A high expression of several hnRNPs is observed in prostate cancers compared to that in benign prostate tissues $(10,11)$. Studies have shown that HNRNPK plays a role in the regulation of androgen receptor (AR) expression via transcriptional and posttranscriptional mechanisms (12, 13). Additionally, HNRNPK is found to be co-localized with AR in a complex inside the nucleoplasm, and both proteins are modulated via treatment with the anti-androgen bicalutamide (14). Moreover, a family-based study found that a variant in the regulatory region of $H N R N P A O$ is associated with elevated cancer incidence (15).

The genetic variants of hnRNP genes are thought to influence gene expression; however, it is still unknown whether hnRNP single nucleotide polymorphisms (SNPs) play a role in prostate cancer prognosis. In the present study, we systematically selected haplotype-tagging SNPs (htSNPs) to capture gene-wide common variations, and evaluated their associations with survival outcomes in patients with prostate cancer after receiving ADT.

\section{Patients and Methods}

Patient and response evaluation. The study population comprised 630 Taiwanese patients with advanced prostate cancer receiving ADT in three medical centres in Taiwan: Kaohsiung Medical University Hospital, Kaohsiung Veterans General Hospital, and National Taiwan University Hospital. The patient inclusion and exclusion criteria have been described previously $(16,17)$. Detailed clinicopathological information was obtained from the patient medical records. The cause and date of death were obtained by linking the personal identification number with the National Death Registry via the Ministry of Health and Welfare, Taiwan. Overall survival (OS) was defined as the time from the initiation of ADT to death from any disease. Cancer-specific survival (CSS) was defined as the duration from the initiation of ADT until death due to cancer. Progression-free survival (PFS) was defined as the time from ADT initiation to tumour progression or cancer-related death. This study was approved by the institutional review board of Kaohsiung Medical University Hospital (KMU HIRB-2013132), and was conducted in accordance with the Good Clinical Practice guidelines. All patients provided written informed consent prior to the study enrolment.

SNP selection and genotyping. The htSNPs within $23 \mathrm{hnRNP}$ genes and their 5-kb flanking regions were obtained from the 1000 Genomes data for Han Chinese in Beijing, China, and Southern Han Chinese with a threshold of minor allele frequency (MAF) of at least 0.03 and an $r^{2}$ threshold of 0.8 (18). Genomic DNA was extracted from the peripheral blood, and the Affymetrix Axiom Genotyping Arrays system (Thermo Fisher Scientific, Waltham, MA, USA) was used for genotyping at the National Centre for Genome Medicine, Taiwan, as described previously (19). Any htSNPs that showed a genotyping call rate of $<0.9$, MAF of $<0.02$, and a Hardy-Weinberg equilibrium of $<0.001$ was excluded, leaving $78 \mathrm{htSNPs}$ for further analyses.

Bioinformatics analysis. We performed functional annotation of the identified htSNPs using HaploReg v4.1 (20). The expression quantitation trait loci analysis between identified htSNPs and PTBP1 gene expression levels was evaluated using HapMap3 lymphoblastoid cell data (21). Differential expression of PTBP1 mRNA in prostate cancer tissues and adjacent normal tissues was analysed using the Oncomine database (22) and The Cancer Genome Atlas Prostate Adenocarcinoma (TCGA PRAD) data (23).

Statistical analysis. Statistical analyses were performed using the SPSS software version 19.0.0 (IBM, Armonk, NY, USA). A twosided $p<0.05$ was considered significant, and multiple testing correction procedures and $q$ values (24) were also applied to report the false discovery rate with a threshold of 0.05 . Meta-analysis of gene expression studies on prostate cancer was expressed as standardized mean difference (SMD), and combined with a model of random effects using RevMan 5.4.1 (Cochrane, London, UK). 
Table I. Clinicopathologic characteristics of the study population.

\begin{tabular}{|c|c|c|c|c|c|c|c|}
\hline \multirow[t]{2}{*}{ Characteristics } & \multirow[t]{2}{*}{$\mathrm{n}(\%)$} & \multicolumn{2}{|c|}{$\mathrm{OS}^{\mathrm{a}}$} & \multicolumn{2}{|c|}{$\mathrm{CSS}^{\mathrm{a}}$} & \multicolumn{2}{|c|}{ PFS $^{a}$} \\
\hline & & HR $(95 \%$ CI $)$ & $p$-Value & HR $(95 \%$ CI $)$ & $p$-Value & HR $(95 \%$ CI $)$ & $p$-Value \\
\hline Total, n (\%) & 630 & $414(65.7)$ & & $314(49.8)$ & & $518(82.6)$ & \\
\hline \multicolumn{8}{|c|}{ Age at diagnosis, years } \\
\hline Median (IQR) & $73(67-79)$ & & & & & & \\
\hline$<74$ & $344(54.7)$ & 1.00 & & 1.00 & & 1.00 & \\
\hline$\geq 74$ & $285(45.3)$ & $1.67(1.37-2.03)$ & $<0.001$ & $1.32(1.06-1.65)$ & 0.014 & $0.72(0.61-0.86)$ & $<0.001$ \\
\hline \multicolumn{8}{|c|}{ PSA at ADT initiation, $\mathrm{ng} / \mathrm{ml}$} \\
\hline Median (IQR) & $34.5(11.25-129)$ & & & & & & \\
\hline$<35$ & $307(50.6)$ & 1.00 & & 1.00 & & 1.00 & \\
\hline$\geq 35$ & $300(49.4)$ & $1.99(1.63-2.44)$ & $<0.001$ & $2.31(1.83-2.91)$ & $<0.001$ & $1.21(1.02-1.45)$ & 0.030 \\
\hline \multicolumn{8}{|l|}{ PSA nadir, ng/ml } \\
\hline Median (IQR) & $0.14(0.01-1.16)$ & & & & & & \\
\hline$<0.15$ & $314(50.7)$ & 1.00 & & 1.00 & & 1.00 & \\
\hline$\geq 0.15$ & $305(49.3)$ & $2.95(2.41-3.61)$ & $<0.001$ & $3.57(2.82-4.53)$ & $<0.001$ & $2.48(2.07-2.96)$ & $<0.001$ \\
\hline \multicolumn{8}{|c|}{ Time to PSA nadir, months } \\
\hline Median (IQR) & $11(5-20)$ & & & & & & \\
\hline$<12$ & $323(52.2)$ & 1.00 & & 1.00 & & 1.00 & \\
\hline$\geq 12$ & $296(47.8)$ & $0.66(0.54-0.80)$ & $<0.001$ & $0.57(0.45-0.71)$ & $<0.001$ & $0.36(0.31-0.44)$ & $<0.001$ \\
\hline \multicolumn{8}{|c|}{ Clinical stage at diagnosis } \\
\hline $\mathrm{T} 1 / \mathrm{T} 2$ & $187(29.9)$ & 1.00 & & 1.00 & & 1.00 & \\
\hline $\mathrm{T} 3 / \mathrm{T} 4 / \mathrm{N} 1$ & $205(32.8)$ & $1.07(0.82-1.39)$ & 0.642 & $1.08(0.79-1.49)$ & 0.636 & $0.86(0.69-1.08)$ & 0.190 \\
\hline M1 & $233(37.3)$ & $2.51(1.97-3.19)$ & $<0.001$ & $3.10(2.33-4.11)$ & $<0.001$ & $1.41(1.14-1.74)$ & 0.002 \\
\hline \multicolumn{8}{|c|}{ Gleason score at diagnosis } \\
\hline $2-6$ & $188(30.6)$ & 1.00 & & 1.00 & & 1.00 & \\
\hline 7 & $194(31.6)$ & $1.07(0.82-1.38)$ & 0.640 & $1.06(0.78-1.44)$ & 0.694 & $1.16(0.93-1.46)$ & 0.189 \\
\hline $8-10$ & $232(37.8)$ & $1.96(1.54-2.48)$ & $<0.001$ & $2.14(1.63-2.82)$ & $<0.001$ & $1.48(1.19-1.84)$ & $<0.001$ \\
\hline
\end{tabular}

OS: Overall survival; CSS: cancer-specific survival; PFS: progression-free survival; IQR: interquartile range; PSA: prostate-specific antigen; HR: hazard ratio; CI: confidence interval. aWith a median follow-up of 165.8 months. Subtotals do not sum to 630 due to missing data.

\section{Results}

Study participant characteristics. The clinical characteristics of the study population are summarized in Table I. In total, $414(65.7 \%)$ patients died, $314(49.8 \%)$ patients died from cancer, and $518(82.6 \%)$ showed disease progression with a median follow-up time of 165.8 months. The age, stage, Gleason score at diagnosis, prostate-specific antigen (PSA) level at ADT initiation, PSA nadir, and time to PSA nadir displayed significant associations with OS, CSS, and PFS in patients with advanced prostate cancer treated with ADT.

Association between hnRNP SNPs and the clinical response to $A D T$ in patients with prostate cancer. The associations between each candidate htSNP and the three clinical outcomes are presented in Table II. Two PTBP1 htSNPs called rs10420407 and rs11549883 that were in strong linkage disequilibrium $\left(r^{2}=0.98\right)$, showed significant associations $(q<0.05)$ with OS following ADT. PTBP1 rs10420407 was the most significant SNP, and patients carrying the A allele had a worse OS than those with a GG genotype [hazard ratio $(\mathrm{HR})=1.96,95 \%$ confidence interval $(\mathrm{CI})=1.42-2.71, p=0.000038$, Table III and
Figure 1A). Multivariate analysis results were consistent with this association after adjusting for age, stage, Gleason score at diagnosis, PSA at ADT initiation, PSA nadir, and time to PSA nadir $(\mathrm{HR}=1.64,95 \% \mathrm{CI}=1.18-2.30, p=0.004$, Table III). Notably, patients carrying the A allele of PTBP1 rs10420407 also had a worse CSS (via univariate analysis) and PFS (via both univariate and multivariate analyses) than those with a GG genotype (Table III and Figure 1B and C).

Functional analyses of PTBPI variants in prostate cancer. To investigate the functional importance of PTBP1 rs10420407 in prostate cancer progression, we first performed functional annotation using HaploReg. The analysis showed that rs10420407 and rs11549883 were situated in regulatory regions associated with active enhancers, DNase hypersensitivity, and RNA polymerase II binding, which indicated a high likelihood of affecting PTBPI gene expression (Table IV). Using HapMap3 lymphoblastoid cell data, a suggested association with PTBP1 expression was observed in rs10420407, with the risk variant allele $\mathrm{A}$, associated with higher $P T B P 1$ expression ( $p=0.076$, Figure 2A). By analysing a TCGA PRAD dataset, we further evaluated the prognostic value of $P T B P 1$ in patients 
Table II. HnRNP SNPs and the p-values of their associations with OS, CSS, and PFS in patients with prostate cancer treated with ADT.

\begin{tabular}{|c|c|c|c|c|c|c|c|c|c|c|c|c|}
\hline \multirow[t]{2}{*}{ Gene } & \multirow[t]{2}{*}{ SNP ID } & \multirow[t]{2}{*}{ Chromosome } & \multirow[t]{2}{*}{ Position } & \multirow[t]{2}{*}{ Allele } & \multirow[t]{2}{*}{ MAF } & \multirow[t]{2}{*}{ HWE } & \multicolumn{2}{|c|}{ OS } & \multicolumn{2}{|c|}{ CSS } & \multicolumn{2}{|c|}{ PFS } \\
\hline & & & & & & & $p$-Value & $q$-Value & $p$-Value & $q$-Value & $p$-Value & $q$-Value \\
\hline FUS & rs3764326 & 16 & 31179217 & $\mathrm{~A}>\mathrm{G}$ & 0.079 & 0.788 & 0.344 & 0.898 & 0.179 & 0.674 & 0.478 & 0.811 \\
\hline FUS & rs741810 & 16 & 31182621 & $\mathrm{C}>\mathrm{A}$ & 0.259 & 0.301 & 0.196 & 0.879 & 0.054 & 0.456 & 0.431 & 0.811 \\
\hline FUS & rs1052352 & 16 & 31183958 & $\mathrm{~T}>\mathrm{C}$ & 0.262 & 0.304 & 0.261 & 0.898 & 0.083 & 0.526 & 0.460 & 0.811 \\
\hline FUS & rs750606880 & 16 & 31183959 & $\mathrm{~T}>\mathrm{C}$ & 0.264 & 0.527 & 0.270 & 0.898 & 0.078 & 0.526 & 0.451 & 0.811 \\
\hline FUS & rs 2735393 & 16 & 31184784 & $\mathrm{~T}>\mathrm{C}$ & 0.260 & 0.300 & 0.200 & 0.879 & 0.052 & 0.456 & 0.443 & 0.811 \\
\hline HNRNPA1L2 & rs146376004 & 13 & 52637720 & $\mathrm{G}>\mathrm{T}$ & 0.039 & 0.062 & 0.671 & 0.898 & 0.454 & 0.829 & 0.035 & 0.341 \\
\hline HNRNPA2B1 & rs2040786 & 7 & 26169116 & $\mathrm{~A}>\mathrm{G}$ & 0.309 & 0.136 & 0.929 & 0.927 & 0.516 & 0.829 & 0.350 & 0.811 \\
\hline HNRNPA2B1 & rs141755540 & 7 & 26169811 & $\mathrm{~A}>\mathrm{G}$ & 0.021 & 1.000 & 0.189 & 0.879 & 0.409 & 0.829 & 0.462 & 0.811 \\
\hline HNRNPA2B1 & rs74646772 & 7 & 26189956 & $\mathrm{G}>\mathrm{A}$ & 0.033 & 1.000 & 0.134 & 0.830 & 0.048 & 0.456 & 0.903 & 0.992 \\
\hline HNRNPA2B1 & rs78391041 & 7 & 26202689 & $\mathrm{~A}>\mathrm{G}$ & 0.028 & 0.078 & 0.713 & 0.898 & 0.522 & 0.829 & 0.724 & 0.956 \\
\hline HNRNPA3 & rs117728932 & 2 & 177209919 & $\mathrm{G}>\mathrm{A}$ & 0.022 & 1.000 & 0.121 & 0.814 & 0.098 & 0.552 & 0.168 & 0.775 \\
\hline HNRNPA3 & rs117087446 & 2 & 177215407 & $\mathrm{~T}>\mathrm{C}$ & 0.049 & 0.652 & 0.418 & 0.898 & 0.119 & 0.582 & 1.000 & 1.000 \\
\hline HNRNPAB & rs2913817 & 5 & 178203039 & $\mathrm{G}>\mathrm{A}$ & 0.367 & 0.493 & 0.541 & 0.898 & 0.641 & 0.836 & 0.975 & 1.000 \\
\hline HNRNPAB & rs9329138 & 5 & 178203912 & $\mathrm{G}>\mathrm{A}$ & 0.181 & 0.788 & 0.715 & 0.898 & 0.423 & 0.829 & 0.297 & 0.811 \\
\hline$H N R N P A B$ & rs146427797 & 5 & 178207804 & $\mathrm{C}>\mathrm{T}$ & 0.021 & 1.000 & 0.787 & 0.898 & 0.896 & 0.836 & 0.446 & 0.811 \\
\hline HNRNPAB & rs142221682 & 5 & 178207995 & $\mathrm{G}>\mathrm{T}$ & 0.022 & 1.000 & 0.759 & 0.898 & 0.595 & 0.836 & 0.219 & 0.811 \\
\hline HNRNPAB & rs2913751 & 5 & 178210446 & $\mathrm{G}>\mathrm{A}$ & 0.452 & 0.421 & 0.405 & 0.898 & 0.721 & 0.836 & 0.179 & 0.775 \\
\hline$H N R N P A B$ & rs3749815 & 5 & 178213284 & $\mathrm{G}>\mathrm{T}$ & 0.116 & 0.160 & 0.589 & 0.898 & 0.158 & 0.674 & 0.532 & 0.864 \\
\hline$H N R N P C$ & rs1243369 & 14 & 21205625 & $\mathrm{C}>\mathrm{T}$ & 0.121 & 0.851 & 0.048 & 0.710 & 0.340 & 0.829 & 0.656 & 0.931 \\
\hline$H N R N P C$ & rs1243370 & 14 & 21206055 & $\mathrm{~T}>\mathrm{C}$ & 0.056 & 0.713 & 0.739 & 0.898 & 0.862 & 0.836 & 0.367 & 0.811 \\
\hline$H N R N P C$ & rs28692643 & 14 & 21223379 & $\mathrm{C}>\mathrm{T}$ & 0.079 & 0.165 & 0.783 & 0.898 & 0.668 & 0.836 & 0.885 & 0.992 \\
\hline$H N R N P C$ & rs76303947 & 14 & 21229508 & $\mathrm{C}>\mathrm{T}$ & 0.029 & 0.421 & 0.732 & 0.898 & 0.845 & 0.836 & 0.546 & 0.865 \\
\hline$H N R N P C$ & rs34514090 & 14 & 21233170 & $\mathrm{G}>\mathrm{A}$ & 0.100 & 1.000 & 0.550 & 0.898 & 0.753 & 0.836 & 0.728 & 0.956 \\
\hline$H N R N P C$ & rs4982433 & 14 & 21234963 & $\mathrm{C}>\mathrm{T}$ & 0.079 & 0.414 & 0.803 & 0.903 & 0.588 & 0.836 & 0.893 & 0.992 \\
\hline$H N R N P C$ & rs117010645 & 14 & 21238823 & $\mathrm{G}>\mathrm{A}$ & 0.024 & 1.000 & 0.080 & 0.710 & 0.024 & 0.399 & 0.924 & 0.997 \\
\hline HNRNPC & rs58422010 & 14 & 21242797 & $\mathrm{~A}>\mathrm{C}$ & 0.217 & 0.412 & 0.321 & 0.898 & 0.353 & 0.829 & 0.865 & 0.992 \\
\hline$H N R N P C$ & rs17197037 & 14 & 21257495 & $A>G$ & 0.313 & 1.000 & 0.729 & 0.898 & 0.526 & 0.829 & 0.351 & 0.811 \\
\hline$H N R N P D$ & rs 149257870 & 4 & 82348236 & $\mathrm{~T}>\mathrm{C}$ & 0.047 & 0.390 & 0.511 & 0.898 & 0.975 & 0.859 & 0.459 & 0.811 \\
\hline$H N R N P D$ & rs117269275 & 4 & 82358326 & $\mathrm{C}>\mathrm{T}$ & 0.079 & 0.577 & 0.938 & 0.927 & 0.896 & 0.836 & 0.555 & 0.865 \\
\hline$H N R N P D$ & rs3828537 & 4 & 82360321 & $\mathrm{~T}>\mathrm{C}$ & 0.071 & 1.000 & 0.106 & 0.788 & 0.442 & 0.829 & 0.950 & 1.000 \\
\hline$H N R N P D$ & rs6839093 & 4 & 82363773 & $\mathrm{C}>\mathrm{T}$ & 0.034 & 0.524 & 0.434 & 0.898 & 0.840 & 0.836 & 0.129 & 0.719 \\
\hline HNRNPDL & rs79740118 & 4 & 82426938 & $\mathrm{~T}>\mathrm{G}$ & 0.026 & 0.349 & 0.562 & 0.898 & 0.396 & 0.829 & 0.572 & 0.866 \\
\hline$H N R N P D L$ & rs 6826022 & 4 & 82427825 & $\mathrm{~T}>\mathrm{C}$ & 0.242 & 0.828 & 0.418 & 0.898 & 0.454 & 0.829 & 0.276 & 0.811 \\
\hline$H N R N P D L$ & rs8732 & 4 & 82430668 & $\mathrm{~A}>\mathrm{G}$ & 0.110 & 0.022 & 0.690 & 0.898 & 0.950 & 0.859 & 0.776 & 0.990 \\
\hline$H N R N P F$ & rs12220230 & 10 & 43395827 & $\mathrm{G}>\mathrm{A}$ & 0.199 & 0.382 & 0.854 & 0.918 & 0.850 & 0.836 & 0.457 & 0.811 \\
\hline$H N R N P F$ & rs148243209 & 10 & 43408284 & $\mathrm{C}>\mathrm{T}$ & 0.026 & 1.000 & 0.783 & 0.898 & 0.261 & 0.829 & 0.691 & 0.956 \\
\hline HNRNPH1 & rs75662200 & 5 & 179610039 & $\mathrm{~A}>\mathrm{G}$ & 0.028 & 1.000 & 0.374 & 0.898 & 0.366 & 0.829 & 0.867 & 0.992 \\
\hline HNRNPHI & rs114666815 & 5 & 179624215 & $\mathrm{G}>\mathrm{A}$ & 0.033 & 1.000 & 0.830 & 0.918 & 0.832 & 0.836 & 1.000 & 1.000 \\
\hline HNRNPH1 & rs117747430 & 5 & 179625095 & $\mathrm{G}>\mathrm{A}$ & 0.029 & 0.421 & 0.551 & 0.898 & 0.725 & 0.836 & 1.000 & 1.000 \\
\hline HNRNPHI & rs6601062 & 5 & 179638641 & $\mathrm{C}>\mathrm{T}$ & 0.029 & 0.420 & 0.627 & 0.898 & 0.268 & 0.829 & 0.095 & 0.671 \\
\hline HNRNPH3 & rs 16925337 & 10 & 68337008 & $\mathrm{G}>\mathrm{T}$ & 0.031 & 1.000 & 0.916 & 0.927 & 0.552 & 0.836 & 0.468 & 0.811 \\
\hline HNRNPH3 & rs2273902 & 10 & 68341899 & $\mathrm{~A}>\mathrm{G}$ & 0.219 & 0.907 & 0.680 & 0.898 & 0.964 & 0.859 & 0.896 & 0.992 \\
\hline HNRNPH3 & rs148528989 & 10 & 68345619 & $\mathrm{~T}>\mathrm{G}$ & 0.029 & 0.419 & 0.086 & 0.710 & 0.899 & 0.836 & 0.360 & 0.811 \\
\hline$H N R N P K$ & rs296892 & 9 & 83982891 & $\mathrm{~A}>\mathrm{G}$ & 0.388 & 0.737 & 0.080 & 0.710 & 0.120 & 0.582 & 0.436 & 0.811 \\
\hline HNRNPL & rs2278010 & 19 & 38837505 & $\mathrm{~A}>\mathrm{C}$ & 0.241 & 0.448 & 0.556 & 0.898 & 0.286 & 0.829 & 0.015 & 0.211 \\
\hline$H N R N P L$ & rs7255774 & 19 & 38844634 & $\mathrm{~T}>\mathrm{C}$ & 0.412 & 0.742 & 0.964 & 0.927 & 0.447 & 0.829 & 0.377 & 0.811 \\
\hline$H N R N P L$ & rs 80215044 & 19 & 38848445 & $\mathrm{C}>\mathrm{T}$ & 0.137 & 0.867 & 0.250 & 0.898 & 0.680 & 0.836 & 0.334 & 0.811 \\
\hline HNRNPLL & rs 438564 & 2 & 38601695 & $\mathrm{G}>\mathrm{A}$ & 0.425 & 0.935 & 0.975 & 0.927 & 0.571 & 0.836 & 0.178 & 0.775 \\
\hline HNRNPLL & rs117984749 & 2 & 38603584 & $\mathrm{~A}>\mathrm{C}$ & 0.053 & 0.693 & 0.672 & 0.898 & 1.000 & 0.870 & 0.006 & 0.211 \\
\hline HNRNPLL & rs67772386 & 2 & 38604246 & $\mathrm{G}>\mathrm{A}$ & 0.244 & 0.829 & 0.872 & 0.924 & 0.811 & 0.836 & 0.431 & 0.811 \\
\hline$H N R N P M$ & rs17160491 & 19 & 8448056 & $\mathrm{~T}>\mathrm{G}$ & 0.483 & 0.811 & 0.645 & 0.898 & 0.716 & 0.836 & 0.424 & 0.811 \\
\hline HNRNPM & rs75883730 & 19 & 8482906 & $\mathrm{G}>\mathrm{A}$ & 0.128 & 1.000 & 0.395 & 0.898 & 0.592 & 0.836 & 0.484 & 0.811 \\
\hline$H N R N P R$ & rs16828286 & 1 & 23318962 & $\mathrm{G}>\mathrm{A}$ & 0.034 & 0.524 & 0.780 & 0.898 & 0.913 & 0.837 & 0.825 & 0.992 \\
\hline$H N R N P R$ & rs77427052 & 1 & 23318978 & $\mathrm{C}>\mathrm{T}$ & 0.037 & 1.000 & 0.201 & 0.879 & 0.294 & 0.829 & 0.577 & 0.866 \\
\hline$H N R N P R$ & rs2103911 & 1 & 23341258 & $\mathrm{C}>\mathrm{A}$ & 0.421 & 0.807 & 0.654 & 0.898 & 0.834 & 0.836 & 0.331 & 0.811 \\
\hline$H N R N P U$ & rs 4412579 & 1 & 244839869 & $\mathrm{~A}>\mathrm{G}$ & 0.038 & 1.000 & 0.079 & 0.710 & 0.490 & 0.829 & 0.933 & 0.997 \\
\hline
\end{tabular}


Table II. Continued

\begin{tabular}{|c|c|c|c|c|c|c|c|c|c|c|c|c|}
\hline \multirow[t]{2}{*}{ Gene } & \multirow[t]{2}{*}{ SNP ID } & \multirow[t]{2}{*}{ Chromosome } & \multirow[t]{2}{*}{ Position } & \multirow[t]{2}{*}{ Allele } & \multirow[t]{2}{*}{ MAF } & \multirow[t]{2}{*}{ HWE } & \multicolumn{2}{|c|}{ OS } & \multicolumn{2}{|c|}{ CSS } & \multicolumn{2}{|c|}{ PFS } \\
\hline & & & & & & & $p$-Value & $q$-Value & $p$-Value & $q$-Value & $p$-Value & $q$-Value \\
\hline HNRNPU & rs12564642 & 1 & 244850055 & $\mathrm{C}>\mathrm{T}$ & 0.023 & 1.000 & 0.938 & 0.927 & 0.800 & 0.836 & 0.056 & 0.482 \\
\hline$H N R N P U$ & rs3766527 & 1 & 244853833 & $\mathrm{~T}>\mathrm{C}$ & 0.104 & 0.666 & 0.494 & 0.898 & 0.691 & 0.836 & 0.013 & 0.211 \\
\hline$H N R N P U$ & rs75053711 & 1 & 244856718 & $\mathrm{~T}>\mathrm{G}$ & 0.085 & 1.000 & 0.506 & 0.898 & 0.373 & 0.829 & 0.872 & 0.992 \\
\hline$H N R N P U$ & rs79923190 & 1 & 244862204 & $\mathrm{~T}>\mathrm{C}$ & 0.024 & 1.000 & 0.307 & 0.898 & 0.052 & 0.456 & 0.787 & 0.990 \\
\hline$H N R N P U$ & rs 1532396 & 1 & 244865889 & $\mathrm{~T}>\mathrm{C}$ & 0.172 & 0.889 & 0.284 & 0.898 & 0.170 & 0.674 & 0.264 & 0.811 \\
\hline$P C B P 1$ & rs7578666 & 2 & 70086436 & $\mathrm{G}>\mathrm{A}$ & 0.261 & 0.918 & 0.687 & 0.898 & 0.285 & 0.829 & 0.363 & 0.811 \\
\hline$P C B P 2$ & rs148880985 & 12 & 53466513 & $\mathrm{G}>\mathrm{A}$ & 0.030 & 1.000 & 0.638 & 0.898 & 0.779 & 0.836 & 0.736 & 0.956 \\
\hline PCBP2 & rs17099611 & 12 & 53473995 & $\mathrm{~A}>\mathrm{G}$ & 0.059 & 0.468 & 0.975 & 0.927 & 0.496 & 0.829 & 0.343 & 0.811 \\
\hline PTBP1 & rs12611349 & 19 & 801820 & $\mathrm{C}>\mathrm{T}$ & 0.029 & 0.086 & 0.621 & 0.898 & 0.764 & 0.836 & 0.125 & 0.719 \\
\hline PTBP1 & rs 10420407 & 19 & 803945 & $\mathrm{G}>\mathrm{A}$ & 0.039 & 0.617 & 0.000038 & 0.003 & 0.004 & 0.143 & 0.013 & 0.211 \\
\hline PTBP1 & rs11549883 & 19 & 810573 & $\mathrm{C}>\mathrm{A}$ & 0.038 & 1.000 & 0.000069 & 0.003 & 0.006 & 0.143 & 0.016 & 0.211 \\
\hline РTBP2 & rs35387107 & 1 & 96735871 & $\mathrm{~A}>\mathrm{C}$ & 0.453 & 0.471 & 0.213 & 0.880 & 0.488 & 0.829 & 0.445 & 0.811 \\
\hline PTBP2 & rs140041979 & 1 & 96740322 & $\mathrm{~A}>\mathrm{C}$ & 0.031 & 1.000 & 0.035 & 0.710 & 0.006 & 0.143 & 0.008 & 0.211 \\
\hline РTBP2 & rs75963976 & 1 & 96753225 & $\mathrm{G}>\mathrm{A}$ & 0.086 & 0.037 & 0.398 & 0.898 & 0.845 & 0.836 & 0.590 & 0.868 \\
\hline РТВP2 & rs17115756 & 1 & 96793020 & $\mathrm{C}>\mathrm{T}$ & 0.045 & 0.365 & 0.350 & 0.898 & 0.348 & 0.829 & 0.174 & 0.775 \\
\hline$P T B P 2$ & rs187025124 & 1 & 96805619 & $\mathrm{G}>\mathrm{A}$ & 0.023 & 0.282 & 0.956 & 0.927 & 0.893 & 0.836 & 0.645 & 0.931 \\
\hline РTBP2 & rs6699932 & 1 & 96806900 & $\mathrm{~A}>\mathrm{G}$ & 0.194 & 0.443 & 0.529 & 0.898 & 0.479 & 0.829 & 0.899 & 0.992 \\
\hline РTBP2 & rs622778 & 1 & 96827698 & $\mathrm{~T}>\mathrm{C}$ & 0.349 & 0.930 & 0.190 & 0.879 & 0.164 & 0.674 & 0.488 & 0.811 \\
\hline$R B M X$ & rs59650690 & 23 & 136859996 & $\mathrm{C}>\mathrm{T}$ & 0.077 & 0.002 & 0.623 & 0.898 & 0.408 & 0.829 & 0.117 & 0.719 \\
\hline$R B M X$ & rs186082704 & 23 & 136877007 & $\mathrm{C}>\mathrm{T}$ & 0.021 & 1.000 & 0.850 & 0.918 & 0.865 & 0.836 & 0.699 & 0.956 \\
\hline$R B M X$ & rs2279166 & 23 & 136879545 & $\mathrm{~A}>\mathrm{G}$ & 0.058 & 0.002 & 0.295 & 0.898 & 0.085 & 0.526 & 0.025 & 0.275 \\
\hline SYNCRIP & rs9450307 & 6 & 85634633 & $\mathrm{G}>\mathrm{A}$ & 0.053 & 0.693 & 0.085 & 0.710 & 0.731 & 0.836 & 0.065 & 0.505 \\
\hline
\end{tabular}

SNP: Single nucleotide polymorphism; OS: overall survival; CSS: cancer-specific survival; PFS: progression-free survival; ADT: androgen deprivation therapy; MAF: minor alleles frequency; HWE: Hardy-Weinberg equilibrium.

Table III. The association of PTBP1 rs 10420407 with OS, CSS, and PFS in prostate cancer patients receiving ADT.

\begin{tabular}{lccccccccc}
\hline Genotype & Frequency & OS & HR $(95 \%$ CI $)$ & $p$-Value & $q$-Value & HR $(95 \% \text { CI })^{\mathrm{a}}$ & $p$-Value & \\
\hline GG/GA/AA & $580 / 49 / 0$ & $371 / 42 / 0$ & $1.96(1.42-2.71)$ & 0.000038 & 0.003 & $1.64(1.18-2.30)$ & 0.004 & & \\
\hline CSS & HR $(95 \%$ CI $)$ & $p$-Value & HR $(95 \% \text { CI })^{\mathrm{a}}$ & $p$-Value & PFS & HR $(95 \%$ CI $)$ & $p$-Value & HR $(95 \% \text { CI })^{\mathrm{a}}$ & $p$-Value \\
\hline $283 / 30 / 0$ & $1.75(1.20-2.56)$ & 0.004 & $1.42(0.95-2.10)$ & 0.085 & $473 / 44 / 0$ & $1.48(1.09-2.02)$ & 0.013 & $1.40(1.02-1.94)$ & 0.039 \\
\hline
\end{tabular}

SNP: Single nucleotide polymorphism; OS: overall survival; CSS: cancer-specific survival; PFS: progression-free survival; ADT: androgen deprivation therapy; HR: hazard ratio; CI: confidence interval. aAdjustment for age, stage, Gleason score at diagnosis, PSA at ADT initiation, PSA nadir, and time to PSA nadir.

with prostate cancer. A higher $P T B P 1$ expression was found in patients with prostate cancers, and the expression was progressively higher as the Gleason score and stage of cancer increased ( $p<0.001$, Figure 2B, C and D). Furthermore, the higher expression of PTBPI not only showed a trend of correlation with poor PFS (Figure 2E), but was significantly correlated with a poor disease-free survival in patients with prostate cancer ( $p=0.034$, Figure $2 \mathrm{~F}$ ). The clinical relevance of $P T B P 1$ was confirmed via meta-analysis of 16 gene expression datasets, comprising a total of 1,152 prostate cancer cases and
326 adjacent normal tissues. $P T B P 1$ in prostate cancers was significantly and consistently up-regulated than in normal tissues (SMD 0.73, 95\% CI=0.45-1.02, $p<0.001$, Figure 2G). These data suggested that PTBPI may be functionally important in the pathogenesis of prostate cancer.

\section{Discussion}

In the present study, we applied multiple strategies, including a haplotype tagging approach to improve SNP coverage, 

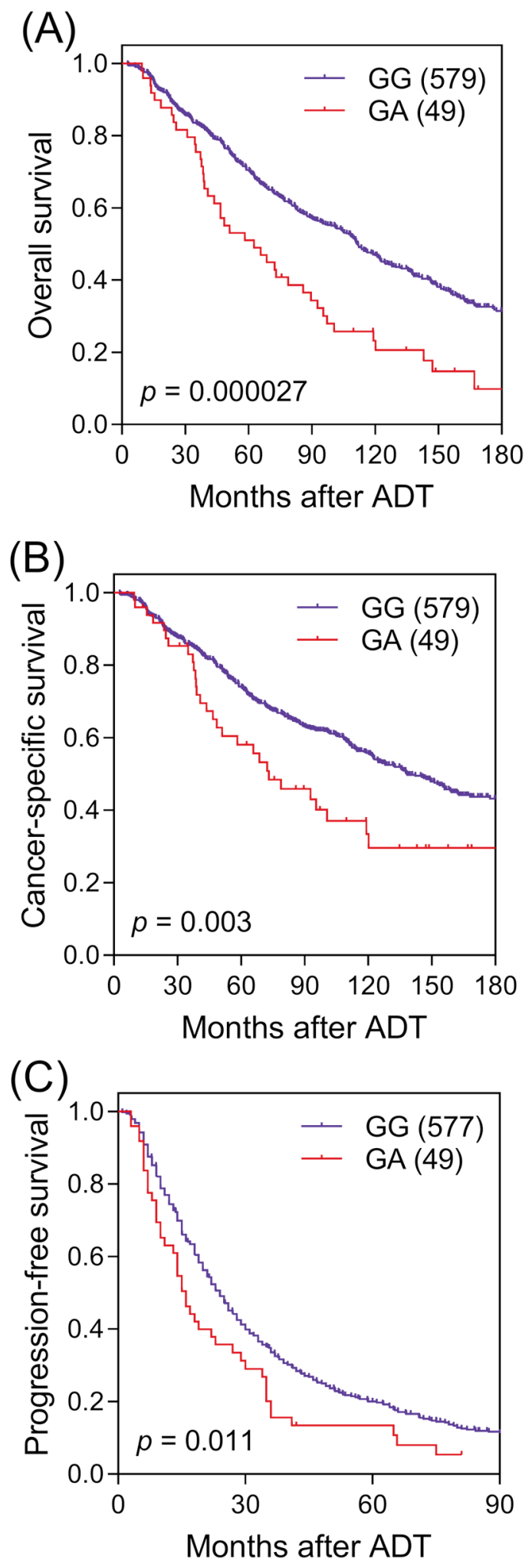

Figure 1. Kaplan-Meier curves for (A) overall survival, (B) cancerspecific survival, and $(C)$ progression-free survival depending on PTBP1 rs 10420407 genotypes. Values in brackets represent the number of patients. ADT: androgen-deprivation therapy. false discovery rate to adjust for multiple comparisons, and meta-analysis and functional annotation to systematically evaluate the clinical significance of hnRNPs in prostate cancer. We found that two htSNPs in PTBPl were associated with poor clinical outcomes in patients with prostate cancer receiving ADT. Furthermore, functional analysis of independent gene expression studies indicated that an increased expression of $P T B P 1$ was correlated with advanced pathological features and worse survival after treatment for prostate cancer. To the best of our knowledge, this is the first report showing the clinical relevance of $P T B P 1$ in the progression of prostate cancer.

According to our genetic association analysis, the strongest signals corresponded to two highly correlated SNPs $\left(r^{2}=0.98\right)$ : rs 10420407, which is an intronic SNP, and rs 11549883, which is a synonymous SNP in an exon of PTBP1. Functional annotation indicated that rs10420407 and rs11549883 were located in a region with histone modifications associated with active transcription of $P T B P 1$, suggesting that these risk loci may influence PTBP1 expression and alter its biological fate. However, additional fine-mapping and functional studies are needed to identify the true causal variants and how they disrupt molecular mechanisms contributing to prostate cancer progression.

PTBP1, belonging to the PTB family of hnRNPs, functions as a regulator of post-transcriptional gene expression by preferentially binding to polypyrimidine-rich stretches of RNA, and is expressed in almost all cell types (25). PTBP1 has been demonstrated to promote cancer progression and regulate apoptosis via several important cancer-associated pathways, such as the epidermal growth factor (26), mitogen-activated protein kinase (27), and phosphoinositide 3-kinase/protein kinase B pathways (28). PTBP1 also regulates hypoxia inducible factor- $1 \alpha$ signalling to alter renal cancer cell migration, invasion, and angiogenesis (29). Moreover, PTBP1 alters pyruvate kinase splicing, leading to an increase in the pyruvate kinase M2 (PKM2)/PKM1 isoform ratio that favours uncoupled glycolysis, a cancer-specific energy metabolism state (9). This PTBP1/PKM2 pathway is found to promote glycolysis, increase cell proliferation, and induce resistance to cell death in multiple tumour types (30). Along with these results, we demonstrated that $P T B P 1$ is up-regulated in prostate cancer and is associated with poor patient prognosis. However, the exact mechanisms of the role of PTBP1 in prostate cancer progression remain to be elucidated.

While this study presents the first comprehensive analysis on the role of genetic variants of hnRNP genes in prostate cancer and the efficacy of ADT, it has certain limitations. First, this was a retrospective study; therefore, the results need to be replicated in prospective clinical trials. Second, the sample size was moderate, and more validation cohorts are required to confirm our findings. Third, we only analysed 
(A)

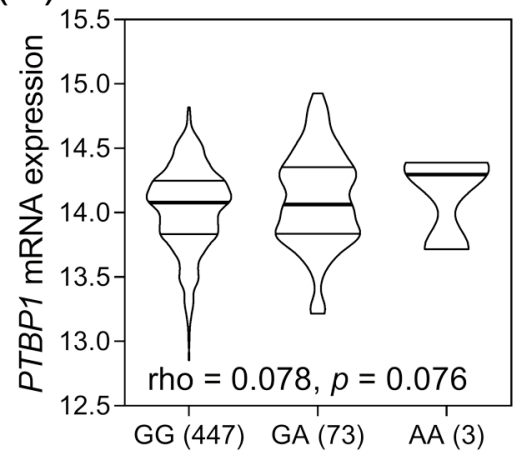

(D)

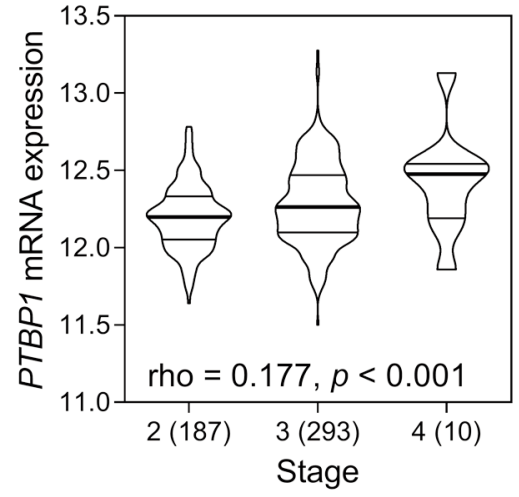

(B)

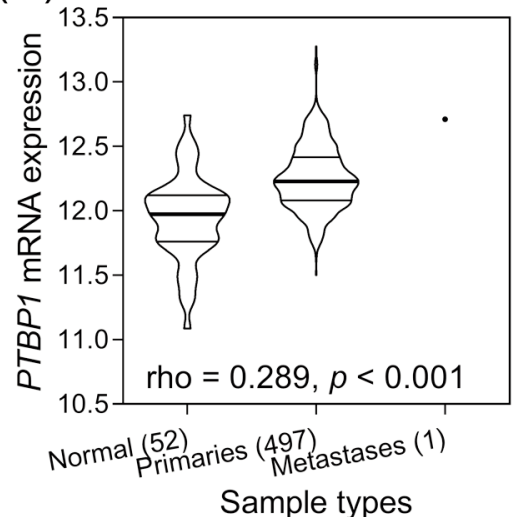

(E)

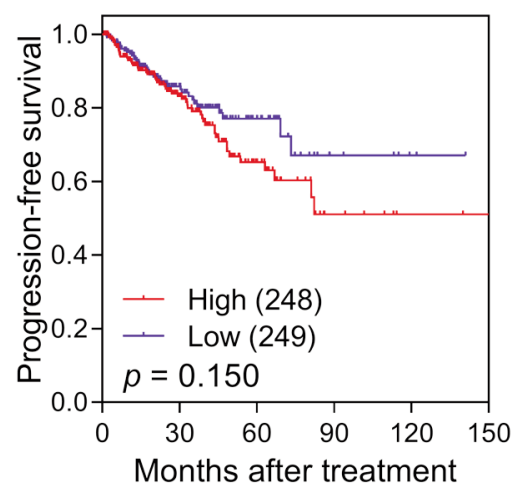

(C)

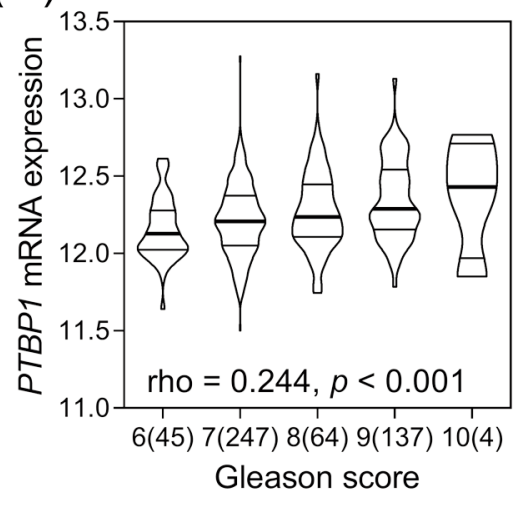

(F)

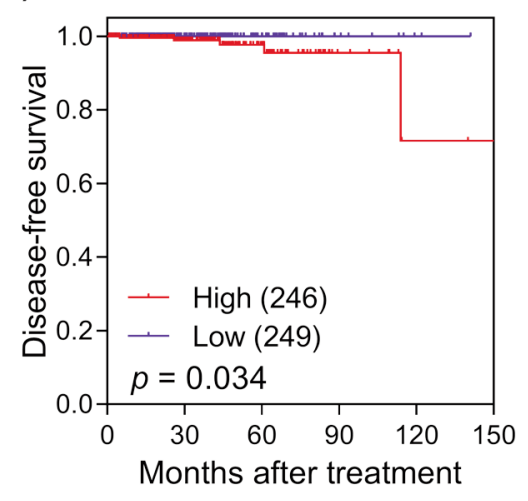

(G)

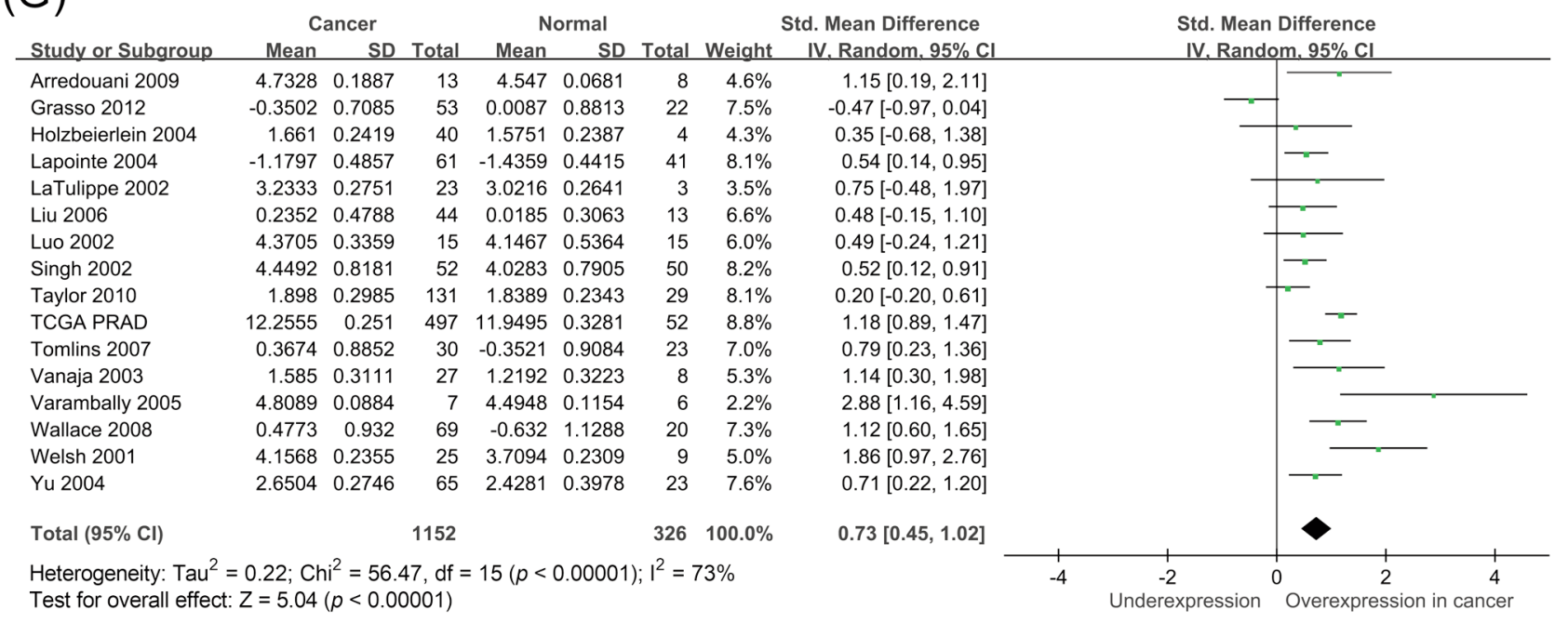

Figure 2. Functional analyses of PTBP1 in prostate cancer. (A) rs10420407 A allele correlates with an increasing PTBP1 expression based on HapMap3 lymphoblastoid cell data. PTBP1 was overexpressed in $(B)$ cancer, $(C)$ high Gleason score, and $(D)$ more advanced stage tumours in TCGA PRAD data. Patients with a high PTBP1 expression tend to have a worse $(E)$ progression-free survival and $(F)$ disease-free survival compared to those with a lower PTBP1 expression. (G) Meta-analysis of 16 studies showed that the expression level of PTBP1 was significantly higher in prostate cancer than in normal tissues. Values in brackets represent the number of patients. TCGA PRAD: The Cancer Genome Atlas Prostate Adenocarcinoma. Rho: Spearman's rank correlation coefficient. SD: standard deviation. IV: inverse variance. CI: confidence interval. 
Table IV. Functional annotation of PTBP1 rs10420407 and rs11549883.

\begin{tabular}{|c|c|c|c|c|c|c|c|c|c|}
\hline SNP ID & Position & $\begin{array}{l}\text { Reference } \\
\text { allele }\end{array}$ & $\begin{array}{c}\text { Alternate } \\
\text { allele }\end{array}$ & $\begin{array}{c}\text { ASN } \\
\text { frequency }\end{array}$ & $\begin{array}{l}\text { Variant } \\
\text { type }\end{array}$ & $\begin{array}{c}\text { Enhancer } \\
\text { histone marks }\end{array}$ & DNAse & $\begin{array}{c}\text { Proteins } \\
\text { bound }\end{array}$ & $\begin{array}{l}\text { Motifs } \\
\text { changed }\end{array}$ \\
\hline rs 10420407 & 803945 & G & A & 0.03 & intronic & & $\begin{array}{c}\text { BLD, BLD, BLD, } \\
\text { ADRL, MUS, } \\
\text { BLD, SKIN }\end{array}$ & POL2 & ZBTB33 \\
\hline rs 11549883 & 810573 & $\mathrm{C}$ & A & 0.02 & synonymous & SKIN, ADRL & $\begin{array}{l}\text { BLD, SKIN, ADRL, } \\
\text { BRN, KID, PLCNT, } \\
\text { OVRY, BLD, LIV, } \\
\text { MUS, BLD, } \\
\text { BRN, SKIN }\end{array}$ & $\begin{array}{c}\text { POL2, } \\
\text { EGR1, } \\
\text { ZBTB33 }\end{array}$ & $\begin{array}{c}\text { ERalpha-a, } \\
\text { HP1-site-factor, } \\
\text { NRSF }\end{array}$ \\
\hline
\end{tabular}

the common genetic variants (MAF>0.03), which might have led to the omission of important low frequency variants. Additionally, all the participants in this study are Taiwanese individuals and these findings may not apply to patients of different ethnicities. Finally, the functional analyses were based on the publically available datasets; additional experiments are needed to elucidate the molecular mechanisms of $P T B P 1$ in prostate cancer.

In conclusion, our study provides evidence that $P T B P 1$ rs10420407 is significantly associated with the efficacy of ADT, and patients with a high expression of PTBPl demonstrate poor survival. Specific genetic variations in conjunction with gene expression and tumour features may help in the selection of patients with prostate cancer who may benefit from ADT or other therapies.

\section{Conflicts of Interest}

The Authors declare that they have no conflicts of interest in regard to this study.

\section{Authors' Contributions}

S-PH, C-YH, C-CY, VCL, and B-YB conceptualized and designed the study. S-PH, L-CC, and B-YB performed the experiments. L$\mathrm{CC}, \mathrm{Y}-\mathrm{TC}, \mathrm{C}-\mathrm{HL}$ and T-LL coordinated and supervised data collection. S-PH, L-CC, Y-TC, and B-YB performed the analysis. All the Authors drafted, reviewed and approved the manuscript.

\section{Acknowledgements}

This work was supported by the Ministry of Science and Technology of Taiwan (grant nos: 108-2314-B-037-029, 108-2314B-037-026-MY2, 108-2320-B-039-050-MY3, 109-2314-B-037-108MY2, and 109-2314-B-037-106-MY3), the Kaohsiung Medical University Hospital (grant nos: KMUH105-5R42, KMUH108-8R53 and KMUH108-8R55), the Kaohsiung Medical University Research Center (grant no: KMU-TC108A04-4), and the China Medical University (grant nos: CMU108-MF-50, CMU109-SR-64, and CMU109-MF-65). The funders had no role in study design, data collection and analysis, decision to publish, or preparation of the manuscript. We thank Chao-Shih Chen for data analysis, and the National Center for Genome Medicine, Ministry of Science and Technology of Taiwan, for technical support. The results published here are based in part on data generated by the HaploReg, HapMap, 1000 Genomes, and TCGA projects.

\section{References}

1 Siegel RL, Miller KD and Jemal A: Cancer statistics, 2020. CA Cancer J Clin 70(1): 7-30, 2020. PMID: 31912902. DOI: 10.3322/caac. 21590

2 Huggins $\mathrm{C}$ and Hodges CV: Studies on prostatic cancer. I. The effect of castration, of estrogen and androgen injection on serum phosphatases in metastatic carcinoma of the prostate. CA Cancer J Clin 22(4): 232-240, 1972. PMID: 4625049. DOI: 10.3322/ canjclin.22.4.232

3 Marques RB, Dits NF, Erkens-Schulze S, van Weerden WM and Jenster G: Bypass mechanisms of the androgen receptor pathway in therapy-resistant prostate cancer cell models. PLoS One 5(10): e13500, 2010. PMID: 20976069. DOI: 10.1371/ journal.pone. 0013500

4 Patel AR and Klein EA: Risk factors for prostate cancer. Nat Clin Pract Urol 6(2): 87-95, 2009. PMID: 19198622. DOI: $10.1038 /$ ncpuro1290

5 Mucci LA, Hjelmborg JB, Harris JR, Czene K, Havelick DJ, Scheike T, Graff RE, Holst K, Möller S, Unger RH, McIntosh C, Nuttall E, Brandt I, Penney KL, Hartman M, Kraft P, Parmigiani G, Christensen K, Koskenvuo M, Holm NV, Heikkilä K, Pukkala E, Skytthe A, Adami HO, Kaprio J and Nordic Twin Study of Cancer (NorTwinCan) Collaboration.: Familial risk and heritability of cancer among twins in Nordic countries. JAMA 315(1): 68-76, 2016. PMID: 26746459. DOI: 10.1001/ jama.2015.17703

6 Krecic AM and Swanson MS: hnRNP complexes: Composition, structure, and function. Curr Opin Cell Biol 11(3): 363-371, 1999. PMID: 10395553. DOI: 10.1016/S0955-0674(99)80051-9

7 Busch A and Hertel KJ: Evolution of SR protein and hnRNP splicing regulatory factors. Wiley Interdiscip Rev RNA 3(1): 112, 2012. PMID: 21898828. DOI: 10.1002/wrna.100

8 Ule J and Blencowe BJ: Alternative splicing regulatory networks: Functions, mechanisms, and evolution. Mol Cell 76(2): 329-345, 2019. PMID: 31626751. DOI: 10.1016/j.molcel.2019.09.017

9 David CJ, Chen M, Assanah M, Canoll P and Manley JL: HnRNP proteins controlled by c-Myc deregulate pyruvate kinase 
mRNA splicing in cancer. Nature 463(7279): 364-368, 2010. PMID: 20010808. DOI: 10.1038/nature08697

10 Fei T, Chen Y, Xiao T, Li W, Cato L, Zhang P, Cotter MB, Bowden M, Lis RT, Zhao SG, Wu Q, Feng FY, Loda M, He HH, Liu XS and Brown M: Genome-wide CRISPR screen identifies HNRNPL as a prostate cancer dependency regulating RNA splicing. Proc Natl Acad Sci USA 114(26): E5207-E5215, 2017. PMID: 28611215. DOI: 10.1073/pnas.1617467114

11 Nagano K, Masters JR, Akpan A, Yang A, Corless S, Wood C, Hastie C, Zvelebil M, Cramer R and Naaby-Hansen S: Differential protein synthesis and expression levels in normal and neoplastic human prostate cells and their regulation by type I and II interferons. Oncogene 23(9): 1693-1703, 2004. PMID: 14647428. DOI: $10.1038 /$ sj.onc. 1207297

12 Mukhopadhyay NK, Kim J, Cinar B, Ramachandran A, Hager MH, Di Vizio D, Adam RM, Rubin MA, Raychaudhuri P, De Benedetti A and Freeman MR: Heterogeneous nuclear ribonucleoprotein $\mathrm{K}$ is a novel regulator of androgen receptor translation. Cancer Res 69(6): 2210-2218, 2009. PMID: 19258514. DOI: 10.1158/0008-5472.CAN-08-2308

13 Wang LG, Johnson EM, Kinoshita Y, Babb JS, Buckley MT, Liebes LF, Melamed J, Liu XM, Kurek R, Ossowski L and Ferrari AC: Androgen receptor overexpression in prostate cancer linked to Pur alpha loss from a novel repressor complex. Cancer Res 68(8): 2678-2688, 2008. PMID: 18413735. DOI: 10.1158/ 0008-5472.CAN-07-6017

14 Barboro P, Repaci E, Ferrari N, Rubagotti A, Boccardo F and Balbi C: Androgen receptor and heterogeneous nuclear ribonucleoprotein $\mathrm{K}$ colocalize in the nucleoplasm and are modulated by bicalutamide and 4-hydroxy-tamoxifen in prostatic cancer cell lines. Prostate 71(13): 1466-1479, 2011. PMID: 21321982. DOI: $10.1002 /$ pros.21366

15 Wei C, Peng B, Han Y, Chen WV, Rother J, Tomlinson GE, Boland CR, Chaussabel D, Frazier ML and Amos CI: Mutations of HNRNPA0 and WIF1 predispose members of a large family to multiple cancers. Fam Cancer 14(2): 297-306, 2015. PMID: 25716654. DOI: 10.1007/s10689-014-9758-8

16 Bao BY, Pao JB, Huang CN, Pu YS, Chang TY, Lan YH, Lu TL, Lee HZ, Juang SH, Chen LM, Hsieh CJ and Huang SP: Polymorphisms inside microRNAs and microRNA target sites predict clinical outcomes in prostate cancer patients receiving androgen-deprivation therapy. Clin Cancer Res 17(4): 928-936, 2011. PMID: 21149617. DOI: 10.1158/1078-0432.CCR-102648

17 Geng JH, Lin VC, Yu CC, Huang CY, Yin HL, Chang TY, Lu TL, Huang SP and Bao BY: Inherited variants in Wnt pathway genes influence outcomes of prostate cancer patients receiving androgen deprivation therapy. Int J Mol Sci 17(12): 1970, 2016. PMID: 27898031. DOI: 10.3390/ijms 17121970

181000 Genomes Project Consortium., Abecasis GR, Auton A, Brooks LD, DePristo MA, Durbin RM, Handsaker RE, Kang HM, Marth GT and McVean GA: An integrated map of genetic variation from 1,092 human genomes. Nature 491(7422): 56-65, 2012. PMID: 23128226. DOI: 10.1038/nature 11632

19 Bao BY, Pao JB, Huang CN, Pu YS, Chang TY, Lan YH, Lu TL, Lee HZ, Chen LM, Ting WC, Hsieh CJ and Huang SP: Significant associations of prostate cancer susceptibility variants with survival in patients treated with androgen-deprivation therapy. Int J Cancer 130(4): 876-884, 2012. PMID: 21445969. DOI: $10.1002 / \mathrm{ijc} .26091$
20 Ward LD and Kellis M: HaploReg v4: Systematic mining of putative causal variants, cell types, regulators and target genes for human complex traits and disease. Nucleic Acids Res 44(D1): D877-D881, 2016. PMID: 26657631. DOI: 10.1093/nar/gkv1340

21 International HapMap Consortium., Frazer KA, Ballinger DG, Cox DR, Hinds DA, Stuve LL, Gibbs RA, Belmont JW, Boudreau A, Hardenbol P, Leal SM, Pasternak S, Wheeler DA, Willis TD, Yu F, Yang H, Zeng C, Gao Y, Hu H, Hu W, Li C, Lin W, Liu S, Pan H, Tang X, Wang J, Wang W, Yu J, Zhang B, Zhang Q, Zhao H, Zhao H, Zhou J, Gabriel SB, Barry R, Blumenstiel B, Camargo A, Defelice M, Faggart M, Goyette M, Gupta S, Moore J, Nguyen H, Onofrio RC, Parkin M, Roy J, Stahl E, Winchester E, Ziaugra L, Altshuler D, Shen Y, Yao Z, Huang W, Chu X, He Y, Jin L, Liu Y, Shen Y, Sun W, Wang H, Wang Y, Wang Y, Xiong X, Xu L, Waye MM, Tsui SK, Xue H, Wong JT, Galver LM, Fan JB, Gunderson K, Murray SS, Oliphant AR, Chee MS, Montpetit A, Chagnon F, Ferretti V, Leboeuf M, Olivier JF, Phillips MS, Roumy S, Sallée C, Verner A, Hudson TJ, Kwok PY, Cai D, Koboldt DC, Miller RD, Pawlikowska L, Taillon-Miller P, Xiao M, Tsui LC, Mak W, Song YQ, Tam PK, Nakamura Y, Kawaguchi T, Kitamoto T, Morizono T, Nagashima A, Ohnishi Y, Sekine A, Tanaka T, Tsunoda T, Deloukas P, Bird CP, Delgado M, Dermitzakis ET, Gwilliam R, Hunt S, Morrison J, Powell D, Stranger BE, Whittaker P, Bentley DR, Daly MJ, de Bakker PI, Barrett J, Chretien YR, Maller J, McCarroll S, Patterson N, Pe'er I, Price A, Purcell S, Richter DJ, Sabeti P, Saxena R, Schaffner SF, Sham PC, Varilly P, Altshuler D, Stein LD, Krishnan L, Smith AV, Tello-Ruiz MK, Thorisson GA, Chakravarti A, Chen PE, Cutler DJ, Kashuk CS, Lin S, Abecasis GR, Guan W, Li Y, Munro HM, Qin ZS, Thomas DJ, McVean G, Auton A, Bottolo L, Cardin N, Eyheramendy S, Freeman C, Marchini J, Myers S, Spencer C, Stephens M, Donnelly P, Cardon LR, Clarke G, Evans DM, Morris AP, Weir BS, Tsunoda T, Mullikin JC, Sherry ST, Feolo M, Skol A, Zhang H, Zeng C, Zhao H, Matsuda I, Fukushima Y, Macer DR, Suda E, Rotimi CN, Adebamowo CA, Ajayi I, Aniagwu T, Marshall PA, Nkwodimmah C, Royal CD, Leppert MF, Dixon M, Peiffer A, Qiu R, Kent A, Kato K, Niikawa N, Adewole IF, Knoppers BM, Foster MW, Clayton EW, Watkin J, Gibbs RA, Belmont JW, Muzny D, Nazareth L, Sodergren E, Weinstock GM, Wheeler DA, Yakub I, Gabriel SB, Onofrio RC, Richter DJ, Ziaugra L, Birren BW, Daly MJ, Altshuler D, Wilson RK, Fulton LL, Rogers J, Burton J, Carter NP, Clee CM, Griffiths M, Jones MC, McLay K, Plumb RW, Ross MT, Sims SK, Willey DL, Chen Z, Han H, Kang L, Godbout M, Wallenburg JC, L'Archevêque P, Bellemare G, Saeki K, Wang H, An D, Fu H, Li Q, Wang Z, Wang R, Holden AL, Brooks LD, McEwen JE, Guyer MS, Wang VO, Peterson JL, Shi M, Spiegel J, Sung LM, Zacharia LF, Collins FS, Kennedy K, Jamieson R and Stewart J: A second generation human haplotype map of over 3.1 million SNPs. Nature 449(7164): 851-861, 2007. PMID: 17943122. DOI: 10.1038/nature06258

22 Rhodes DR, Yu J, Shanker K, Deshpande N, Varambally R, Ghosh D, Barrette T, Pandey A and Chinnaiyan AM: ONCOMINE: A cancer microarray database and integrated datamining platform. Neoplasia 6(1): 1-6, 2004. PMID: 15068665. DOI: $10.1016 / \mathrm{s} 1476-5586(04) 80047-2$

23 Cancer Genome Atlas Research Network.: Comprehensive genomic characterization defines human glioblastoma genes and core pathways. Nature 455(7216): 1061-1068, 2008. PMID: 18772890. DOI: $10.1038 /$ nature07385 
24 Storey JD and Tibshirani R: Statistical significance for genomewide studies. Proc Natl Acad Sci U S A 100(16): 94409445, 2003. PMID: 12883005. DOI: 10.1073/pnas.1530509100

25 Ghetti A, Piñol-Roma S, Michael WM, Morandi C and Dreyfuss G: hnRNP I, the polypyrimidine tract-binding protein: Distinct nuclear localization and association with hnRNAs. Nucleic Acids Res 20(14): 3671-3678, 1992. PMID: 1641332. DOI: $10.1093 /$ nar/20.14.3671

26 Ferrarese R, Harsh GR 4th, Yadav AK, Bug E, Maticzka D, Reichardt W, Dombrowski SM, Miller TE, Masilamani AP, Dai F, Kim H, Hadler M, Scholtens DM, Yu IL, Beck J, Srinivasasainagendra V, Costa F, Baxan N, Pfeifer D, von Elverfeldt D, Backofen R, Weyerbrock A, Duarte CW, He X, Prinz M, Chandler JP, Vogel H, Chakravarti A, Rich JN, Carro MS and Bredel M: Lineage-specific splicing of a brain-enriched alternative exon promotes glioblastoma progression. J Clin Invest 124(7): 2861-2876, 2014. PMID: 24865424. DOI: 10.1172/JCI68836

27 Hollander D, Donyo M, Atias N, Mekahel K, Melamed Z, Yannai S, Lev-Maor G, Shilo A, Schwartz S, Barshack I, Sharan $\mathrm{R}$ and Ast G: A network-based analysis of colon cancer splicing changes reveals a tumorigenesis-favoring regulatory pathway emanating from ELK1. Genome Res 26(4): 541-553, 2016. PMID: 26860615. DOI: 10.1101/gr.193169.115
28 Wang X, Li Y, Fan Y, Yu X, Mao X and Jin F: PTBP1 promotes the growth of breast cancer cells through the PTEN/Akt pathway and autophagy. J Cell Physiol 233(11): 8930-8939, 2018. PMID: 29856478. DOI: $10.1002 /$ jcp. 26823

29 Shan H, Hou P, Zhang M, Li L, Pan Y, Chen F, Jiang T, Bai J and Zheng J: PTBP1 knockdown in renal cell carcinoma inhibits cell migration, invasion and angiogenesis in vitro and metastasis in vivo via the hypoxia inducible factor- $1 \alpha$ pathway. Int $\mathrm{J}$ Oncol 52(5): 1613-1622, 2018. PMID: 29512730. DOI: 10.3892/ijo.2018.4296

30 Desai S, Ding M, Wang B, Lu Z, Zhao Q, Shaw K, Yung WK, Weinstein JN, Tan M and Yao J: Tissue-specific isoform switch and DNA hypomethylation of the pyruvate kinase PKM gene in human cancers. Oncotarget 5(18): 8202-8210, 2014. PMID: 24077665. DOI: $10.18632 /$ oncotarget. 1159

Received February 12, 2021

Revised March 2, 2021

Accepted March 3, 2021 